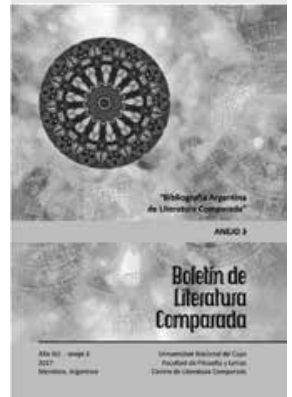

\title{
Boletín de Literatura Comparada. Centro de Literatura Comparada Nicolás J. Dornheim, Universidad Nacional de Cuyo
}

\author{
Paula Simón • \\ CONICET / Universidad Nacional de Cuyo
}

En 1976 se inauguró el Centro de Literatura Comparada en la Facultad de Filosofía y Letras de la Universidad Nacional de Cuyo, bajo la dirección del Dr. Nicolás Jorge Dornheim. Siendo uno de los pioneros en Argentina, este centro se planteó objetivos concretos como la creación de una biblioteca especializada que resolviera el problema de la carencia de material bibliográfico sobre Literatura Comparada disponible en los repositorios nacionales. Con ese mismo espíritu, el CLC se propuso difundir las investigaciones llevadas a cabo por sus miembros, lo que derivó en la edición del Boletín de Literatura Comparada, una actividad que ha acompañado el desarrollo del CLC desde sus inicios.

Con periodicidad anual, el Boletin de Literatura Comparada (ISSN 0325-3775) se ha publicado ininterrumpidamente desde 1976 gracias al apoyo de la Secretaría de Ciencia, Técnica y Posgrado de la Universidad Nacional de Cuyo. Aunque en una primera etapa recogió solo los resultados de las investigaciones de los miembros del Centro de Literatura Comparada, pronto abrió su convocatoria a especialistas en la disciplina del ámbito nacional e internacional. Actualmente, bajo la dirección de la Dra. Lila Bujaldón, en varias oportunidades presidenta y vicepresidenta de la Asociación Argentina de Literatura Comparada (AALC), es una publicación con referato que se obtiene por canje y está indexada en reconocidos índices como el Núcleo Básico de Revistas Científicas Argentinas (CAICYT, CONICET), Latindex y Dialnet.

Entre los contenidos generales del Boletín se destacan artículos y reseñas que se enmarcan en las diversas áreas del quehacer comparatista, tales como la literatura de viaje, la literatura de exilio y migración, la literatura de minorías, las interrelaciones entre la literatura y otras artes, así como entre la literatura y otras disciplinas, las relaciones entre la literatura y las catástrofes históricas, la tematología, la imagología, la recepción, los estudios sobre traducción y autotraducción y la historia de la Literatura Comparada, entre otras. A lo largo de sus páginas es posible consultar artículos originales de especialistas de trayectoria nacional e internacional, tales como Manfred Beller, Jean Bessière, Oscar Caeiro, Rolando Costa Picazo, Hugo

\footnotetext{
- Investigadora Adjunta del Consejo Nacional de Investigaciones Científicas y Técnicas (CONICET) con sede de trabajo en el Centro de Literatura Comparada de la Universidad Nacional de Cuyo. Forma parte del Grupo de Estudios del Exilio Literario (Universidad Autónoma de Barcelona, España). Autora del libro La escritura de las alambradas. Exilio y memoria en los testimonios españoles sobre los campos de concentración franceses (2012).
} 
Dyserinck, Ottmar Ette, María Rosa Lojo y Christoph Rodiek, por citar solo a algunos. Cabe destacar que en los números XXVI-XXVII (2001-2002) y XXXVI (2011) se ofrecen índices generales de la revista.

A partir de 2011 el Boletín incorporó una sección de entrevistas en las cuales se recoge la palabra de importantes especialistas de la disciplina que se desempeñan en variados ámbitos académicos y culturales, tales como Alfredo Luzi (Universidad de Macerata); Margaret W. Ferguson (Universidad de California, Davis); José Antonio Pérez Bowie (Universidad de Salamanca) y Anil Bhatti (Universidad Jawaharlal Nehru). Dichas entrevistas permiten difundir el estado de la cuestión de la Literatura Comparada, como así también los debates vigentes que afronta la disciplina en el contexto internacional.

En varias ocasiones el Boletín de Literatura Comparada ha dado lugar a la publicación de actas de congresos de la Asociación Argentina de Literatura Comparada, como son los casos del número XXXIV (2009) y XXXV (2010), en los cuales se accede a las Actas de las VIII Jornadas Nacionales de Literatura Comparada, celebradas en la Facultad de Filosofía y Letras de la Universidad Nacional de Cuyo en 2007. Asimismo, el Boletín ha editado ensayos presentados en coloquios de la especialidad, tal como lo prueban los números XXVIII al XXX (2003-2005) en homenaje a Nicolás J. Dornheim, que reúnen en forma póstuma los materiales del Coloquio Nacional Literatura de Viaje, en la mencionada Casa de Estudios.

Una de las actividades prioritarias del Centro de Literatura Comparada ha sido el desarrollo del proyecto BIALICO (Bibliografía Argentina de Literatura Comparada), que consiste en la confección de bibliografías detalladas y comentadas del trabajo comparatista llevado a cabo en nuestro país por escritores, críticos, teóricos e historiadores de la literatura. Los resultados de la primera etapa de dicho proyecto fueron difundidos en dos fascículos, anejos del Boletín de Literatura Comparada, que aparecieron en 2001 y 2003-2005. En estos volúmenes se publicó la bibliografía comparatista de intelectuales del siglo XIX y XX que permitió explorar los antecedentes y los referentes del comparatismo nacional. El primer fascículo (2001) relevó el trabajo de Ángel J. Battistessa, Oscar Caeiro, Jorge Dubatti, Delfín Leocadio Garasa, Roberto Federico Giusti, Alma Novella Marani, Arturo Marasso, Pierina Lidia Moreau y Emilia de Zuleta. En tanto, el segundo fascículo (2006) reunió la bibliografía comparatista de Enrique Anderson Imbert, Rafael Alberto Arrieta, Emilio Carilla, Raúl Héctor Castagnino, Guillermo de Torre, Juan María Gutiérrez, María Rosa Lida de Malkiel, Marta Zulema Palermo y Gloria Videla de Rivero.

La segunda etapa del proyecto BIALICO, llevada a cabo entre 2013 y 2015 en el marco de un Proyecto de Investigación Plurianual del Consejo Nacional de Investigaciones Científicas y Técnicas (CONICET), permitió confeccionar nuevas bibliografías de intelectuales argentinos que transitaron enfoques comparatistas en su producción científica, entre los que se cuentan Esteban Echeverría, Ricardo Rojas, Arturo Farinelli, María Teresa Maiorana, Nicolás Jorge Dornheim y Gloria Galli de Ortega. Estas bibliografías han sido publicadas en un tercer fascículo del Boletín de Literatura Comparada (2018), de próxima aparición.

El Boletín de Literatura Comparada ha sido desde el primer número hasta la actualidad, cuando es inminente la aparición del número LXI, un fiel testigo de la vitalidad de la Literatura Comparada tanto en Argentina como en otros ámbitos académicos. Así lo prueba, entre otros hechos, la extensa reseña que mereció en el órgano bibliográfico de la AILC/ICLA, Recherche Littéraire/Literary Research (32, 
2015, 149-152). Aunque se trata de un volumen editado en soporte papel, los índices de los últimos números y los dos anejos de BIALICO correspondientes a la primera etapa del proyecto pueden consultarse en la página web del CLC (http:// ffyl.uncuyo.edu.ar/centro-de-literatura-comparada).

A través de los artículos, reseñas y entrevistas recogidos en el Boletín de Literatura Comparada es posible reflexionar sobre la historia del comparatismo, como así también sobre los temas y problemas que han definido la agenda nacional e internacional de la disciplina. Por este motivo, el Boletín de Literatura Comparada goza de un alto impacto que lo ubica como una publicación de referencia para la comunidad académica de lengua española especializada en Literatura Comparada, logrado entre otros méritos por una continuidad de más de 40 años de existencia.

\section{Simón, Paula}

«Boletín de Literatura Comparada. Centro de Literatura Comparada Nicolás J. Dornheim, Universidad Nacional 